\title{
Preoperative anti-PF4/heparin antibody level predicts adverse outcome after cardiac surgery
}

\author{
Elliott Bennett-Guerrero, MD, ${ }^{a *}$ Thomas F. Slaughter, MD, ${ }^{\mathrm{b} *}$ William D. White, MPH, ${ }^{\mathrm{a}}$ Ian J. Welsby, MD, \\ Charles S. Greenberg, MD, ${ }^{c}$ Habib El-Moalem, PhD, ${ }^{a}$ and Thomas L. Ortel, MD, PhD ${ }^{c}$
}

From the Departments of Anesthesiology ${ }^{\mathrm{a}}$ and Hematology, ${ }^{\mathrm{c}}$ Duke University Medical Center, Durham, NC, and the Department of Anesthesiology, ${ }^{\text {b }}$ Wake Forest University School of Medicine, WinstonSalem, NC.

Supported in part by grant HL03205 from the National Institutes of Health, National Heart, Lung, and Blood Institute (T.F.S.), and departmental funds.

Received for publication Feb 11, 2005; revisions received April 14, 2005; accepted for publication July 29, 2005 .

Address for reprints: Elliott BennettGuerrero, MD, Duke Clinical Research Institute, Duke University (DUMC Box 3094), Durham, NC 27710 (E-mail: elliott. bennettguerrero@duke.edu).

*Drs Bennet-Guerrero and Slaughter contributed equally to this work.

J Thorac Cardiovasc Surg 2005;130:1567-72

$0022-5223 / \$ 30.00$

Copyright $\odot 2005$ by The American Association for Thoracic Surgery

doi:10.1016/j.jtcvs.2005.07.052
Objective: Preexisting serum antibodies to heparin/platelet factor 4 complexes may predispose adult cardiac surgical patients to increased perioperative morbidity and mortality. We sought to determine the association between preoperative serum antibodies directed against platelet factor 4/heparin complexes and major complications (in-hospital death or length of stay $>10$ days) in adult cardiac surgical patients.

Methods: In a prospective observational study of 466 patients undergoing elective coronary artery bypass grafting, valvular heart surgery, or both, preoperative serum was assayed for anti-platelet factor $4 /$ heparin antibody by using a commercially available enzyme-linked immunosorbent assay (Asserachrom HPIA). Known preoperative risk factors were assessed, and patients were assigned a risk score by using the validated method of Parsonnet and colleagues.

Results: Major complications (death or postoperative hospitalization $>10$ days) occurred in 108 patients (23\%). Overall, 59 (13\%) patients had a positive preoperative anti-platelet factor 4/heparin antibody screen (upper limit of normal is 0.5 optical density units). A positive assay result independently predicted an increased risk of major complications $(P=.0284$; odds ratio, 1.98 ; $95 \%$ confidence interval, 1.06-3.62) over and above the effect of the Parsonnet risk score $(P<.001$; odds ratio, 1.07; 95\% confidence interval, 1.05-1.10). The level of preoperative antiplatelet factor 4/heparin antibody was also significantly associated with major complications $(P=.036$; odds ratio, $1.31 ; 95 \%$ confidence interval, 1.02-1.68) independently of the Parsonnet risk score. No association $(P>.75)$ existed between the Parsonnet risk score and preoperative anti-platelet factor $4 /$ heparin antibody level.

Conclusions: Serum antibodies directed against platelet factor 4/heparin complexes are prevalent in the adult patient population undergoing cardiac surgery. The presence of these antibodies before surgery is an independent predictor for death or prolonged hospitalization after adult cardiac surgery.

$\mathrm{M}$ ore than 350,000 adult cardiac operations are performed in the United States annually. Although mortality rates are relatively low, perioperative complications remain common and include myocardial infarction, atrial fibrillation, ventricular failure, and non-cardiac-related morbidities such as renal dysfunction, gastrointestinal dysfunction, acute lung injury, stroke, and infections. There is increasing recognition that many complications in cardiac surgical ${ }^{1,2}$ and other critically ill patients ${ }^{3}$ may result from inflammatory or prothrombotic responses to tissue trauma. A possible prothrombotic trigger may result from the development of serum antibodies directed against platelet factor $4(\mathrm{PF} 4) /$ heparin complexes. ${ }^{4} \mathrm{PF} 4 /$ heparin complexes bound to platelet surfaces act as antibody targets and lead to platelet activation with antibody binding. All adult cardiac 


$$
\begin{aligned}
& \text { Abbreviations and Acronyms } \\
& \text { CI = confidence interval } \\
& \text { ELISA }=\text { enzyme-linked immunosorbent assay } \\
& \text { HIT = heparin-induced thrombocytopenia } \\
& \text { HIT }(\mathrm{T})=\text { heparin-induced thrombocytopenia and } \\
& \text { thrombosis } \\
& \text { Ig = immunoglobulin } \\
& \text { OD = optical density } \\
& \text { OR = odds ratio } \\
& \text { PF4 = platelet factor } 4
\end{aligned}
$$

surgical patients are exposed to heparin at the time of cardiac catheterization or during the subsequent surgical procedure itself. A substantial fraction of these patients have antibodies directed against PF4/heparin complexes, and a small subset progress to having heparin-induced thrombocytopenia (HIT). ${ }^{5}$

HIT comprises a syndrome ranging from the asymptomatic presence of serum antibodies directed against PF4/ heparin complexes to isolated thrombocytopenia and, in the most severe cases, thrombotic complications characterized by venous and/or arterial thromboses that result in limb amputations and death. ${ }^{6,7}$ In most published series to date, antibodies to PF4/heparin complexes have been detected in nearly one third of patients after cardiac surgery. ${ }^{8-12}$ Also, although the incidence of clinically apparent HIT is low $(1 \%-3 \%)$, no large prospective investigations have assessed the relationship between the preoperative presence of antiPF4/heparin antibodies and adverse outcomes after cardiac surgery. Therefore, we tested the hypothesis that preexisting serum antibodies directed against PF4/heparin complexes are associated with in-hospital death or prolonged hospitalization after adult cardiac surgery.

\section{Methods}

\section{Patient Selection}

After institutional review board approval and informed consent, patients undergoing coronary artery bypass grafting surgery, valvular heart surgery, or both, were enrolled in a prospective observational study at Duke University Medical Center in Durham, North Carolina, and the Mount Sinai Medical Center in New York, New York. Given the need for informed consent and the exclusion of patients undergoing emergency operations, we could not enroll consecutive patients.

\section{Protocol}

After benzodiazepine premedication, venous, radial arterial, and central venous catheters were inserted. Induction and maintenance of general anesthesia were accomplished with midazolam hydrochloride and fentanyl citrate infusions. Patients underwent standard nonpulsatile hypothermic $\left(28^{\circ} \mathrm{C}-32^{\circ} \mathrm{C}\right)$ cardiopulmonary bypass with a membrane oxygenator. Porcine heparin sodium (Elkins-Sinn, Inc, Cherry Hill, NJ) was administered as a load of
$300 \mathrm{U} / \mathrm{kg}$ and supplemented as necessary to maintain an activated clotting time of at least 450 seconds during cardiopulmonary bypass. After cardiopulmonary bypass, heparin was neutralized with protamine sulfate (Eli Lilly and Co, Indianapolis, Ind) administered in a dose of $1 \mathrm{mg}$ of protamine to $100 \mathrm{U}$ of heparin. Patients were managed after surgery according to standard institutional cardiac surgery "care maps" in which patients without significant complications were discharged from the hospital on postoperative day 4 or 5 .

Blood samples for the determination of serum anti-PF4/heparin antibody concentrations were obtained through an arterial catheter immediately before the induction of general anesthesia; samples were collected in glass tubes without additives and centrifuged for 10 minutes at $2000 \mathrm{~g}$. Plasma was stored at $-70^{\circ} \mathrm{C}$ until assayed. Levels of anti-PF4/heparin antibodies were measured by using a commercially available enzyme-linked immunosorbent assay (ELISA) according to the manufacturer's instructions (Asserachrom HPIA; Diagnostica Stago, Parsippany, NJ), as described previously. ${ }^{13}$ In brief, antibodies bound to heparin/PF4 complexes on the microtiter plate were measured by using a mixture of antihuman immunoglobulin (Ig) G, IgM, and IgA. A positive antibody screen was defined by the manufacturer as an absorbance value of greater than 0.5 optical density (OD) units. All laboratory measurements were performed on coded samples to blind investigators to the patients' identity and outcome.

\section{Preoperative Risk Scoring and Definitions of Patient Characteristics}

Demographic information was collected before surgery, and a risk score for mortality and morbidity was calculated according to the previously validated Parsonnet risk scoring system. ${ }^{14,15}$ This system sums assigned values for 19 factors known to increase mortality and morbidity after cardiac surgery and generates a score between 0 (ie, no risk factors) and 148; most patients in the original series by Parsonnet and colleagues ${ }^{14}(n=4832)$ received a score of 0 to 30 , and approximately $50 \%$ of patients received a score of 0 to 9 .

Diabetes mellitus was considered present if a patient required preoperative oral hypoglycemic or insulin medication. Myocardial infarction was defined as electrocardiographic evidence of an old myocardial infarction or previously documented episodes of increased creatine kinase MB isoenzymes without concurrent electrocardiographic changes. Hypertension was defined as blood pressure greater than $140 / 90 \mathrm{~mm} \mathrm{Hg}$ documented on at least 3 occasions or a history of increased blood pressure requiring medication. A history of congestive heart failure required at least 1 documented episode of congestive heart failure diagnosed by using standard chest radiograph examination, physical examination, and hemodynamic criteria. A history of stroke was defined as the development of a focal preoperative neurologic deficit. A history of atrial fibrillation was defined as any previous episode of atrial fibrillation.

\section{Outcomes}

Postoperative length of stay was defined as the number of days from the day of operation (day 0) to hospital discharge or death. The major complication rate was the primary outcome of this study and was defined as either in-hospital death or a postoperative length of stay longer than 10 days in survivors. A recent retro- 
TABLE 1. Preoperative patient characteristics

\begin{tabular}{|c|c|c|c|c|}
\hline Variable & All patients & Negative: PF4/heparin $\leq 0.5$ & Positive: PF4/heparin >0.5 & $P$ value \\
\hline No. & 466 & $407(87 \%)$ & $59(13 \%)$ & \\
\hline Age (y) & $67 \pm 11$ & $67 \pm 11$ & $65 \pm 12$ & .2228 \\
\hline Parsonnet risk score & $12 \pm 8$ & $12 \pm 8$ & $12 \pm 8$ & .9971 \\
\hline Ejection fraction* & $0.44 \pm 0.15$ & $0.44 \pm 0.15$ & $0.41 \pm 0.17$ & .2214 \\
\hline Preoperative hematocrit & $0.38 \pm 0.05$ & $0.38 \pm 0.05$ & $0.37 \pm 0.05$ & .2414 \\
\hline Preoperative platelet count & $215 \pm 70$ & $215 \pm 72$ & $214 \pm 58$ & .572 \\
\hline Height $(\mathrm{cm})$ & $169 \pm 10$ & $169 \pm 9$ & $165 \pm 11$ & .0124 \\
\hline Weight $(\mathrm{kg})$ & $77 \pm 16$ & $77 \pm 16$ & $75 \pm 17$ & .3975 \\
\hline Body mass index $\left(\mathrm{kg} / \mathrm{m}^{2}\right)$ & $27 \pm 5$ & $27 \pm 5$ & $28 \pm 7$ & .6735 \\
\hline History of diabetes & $23 \%$ & $23 \%$ & $22 \%$ & .9999 \\
\hline Female sex & $32 \%$ & $31 \%$ & $37 \%$ & .3696 \\
\hline Caucasian & $75 \%$ & $76 \%$ & $63 \%$ & $.0262 \dagger$ \\
\hline Not Caucasian & $25 \%$ & $24 \%$ & $37 \%$ & \\
\hline Preoperative IV heparin & $22 \%$ & $23 \%$ & $17 \%$ & .3207 \\
\hline Preoperative aspirin & $56 \%$ & $56 \%$ & $54 \%$ & .8888 \\
\hline
\end{tabular}

Data are presented as mean \pm SD as appropriate. Percentages are of the total, ie, all 466 patients. $P$ values are from Wilcoxon rank sum tests (continuous measures) or Pearson $\chi^{2}$ tests (categories) for group differences. PF4/heparin, Preoperative serum anti-platelet factor 4/heparin immunoglobulin $\mathrm{G}$ level; $I V$, intravenous. *Preoperative left ventricular ejection fraction. †Refers to comparison for race.

spective analysis of 2609 patients undergoing cardiac surgery at Duke University Medical Center demonstrated that most patients with a length of stay longer than 10 days experienced morbidity, ${ }^{16}$ so a prolonged length of stay ( $>10$ days) was selected as a surrogate for the occurrence of significant medical complications. All outcome measurements were performed by investigators blinded to the patients' laboratory data.

\section{Statistical Analysis}

Given an estimated incidence of $15 \%$ for the primary end point (death or hospital length of stay $>10$ days), a sample size of at least 400 subjects was expected to generate at least 60 events and to allow for a stable logistic regression model to test our hypothesis. The primary hypothesis was that increased preoperative concentrations of serum anti-PF4/heparin antibodies are associated with death or prolonged hospitalization, as defined previously.

Unadjusted statistical analysis consisted of Pearson or Spearman correlation tests between continuous variables, Wilcoxon 2-sample rank sum tests for relating continuous variables with response categories, and contingency table $\chi^{2}$ tests for associations between categorical variables. Adjusted statistical analysis used a multivariable logistic regression model approach that modeled response as a function of the preoperative serum anti-PF4/heparin antibody result and the Parsonnet risk score. The antibody result was categorized as normal or abnormal (an absorbance value $>0.5$ OD units). The validated Parsonnet risk score was always included in the different models to adjust for the effect of preoperative patient comorbidities. This analysis tested the effects of preoperative serum anti-PF4/heparin antibody and Parsonnet risk score independently; each was adjusted for the information contributed by the other. The assumption of linearity of fit in the logit model was validated against nonlinear transformations and cubic spline fits.

We further tested the validity of the observed associations by constructing a multivariable logistic model that included preoperative serum anti-PF4/heparin antibody level as a continu- ous variable (log transformed) along with the Parsonnet risk score. In addition, we used univariate logistic regression to test the association of the perioperative characteristics listed in Table 1 with major complications, adjusting for the Parsonnet risk score. These factors included some variables represented in the preoperative risk score (eg, age), as well as other known risk factors not included (eg, height). Factors demonstrating a significant univariate association $(P<.05)$ with major complications were tested as a block against the hypothesized model to determine whether they would supplant the significance of the preoperative serum antiPF4/heparin antibody result. All statistical calculations and analyses were performed by using the SAS software system (SAS Institute Inc, Cary, NC).

\section{Results}

A total of 466 patients were enrolled. Preoperative demographics and intraoperative characteristics for the study population are presented in Table 1. Of note, only race and height were statistically significantly different between those with increased versus normal antibody assay results.

Preoperative serum anti-PF4/heparin antibody levels ranged from 0.02 to 3.11 OD units, with a mean of 0.29 (SD, \pm 0.37 ; median, 0.17). The Parsonnet preoperative risk score ranged from 0 to 41 , with a mean of 11.9 (SD, \pm 8.4 ; median, 11). Overall, major complication occurred in 108 patients $(23.2 \%)$, as summarized in Table 2. In-hospital death occurred in 23 patients (4.9\%), and 96 patients (20.6\%) had a postoperative hospital length of stay longer than 10 days. A total of 85 patients (18.2\%) remained hospitalized for longer than 10 days and survived to discharge. Overall, the postoperative length of stay ranged from 0 to 180 days, with a mean of 12.0 days (SD, \pm 18.1 days; median, 7 days). Among survivors, the length of stay 
TABLE 2. Intraoperative and postoperative patient characteristics

\begin{tabular}{|c|c|c|c|c|}
\hline Variable & All patients & Negative: PF4/heparin $\leq 0.5$ & Positive: PF4/heparin $>0.5$ & $P$ value \\
\hline No. & 466 & $407(87 \%)$ & $59(13 \%)$ & \\
\hline CABG only & $72 \%$ & $72 \%$ & $70 \%$ & $.8983 \dagger$ \\
\hline Valve only & $24 \%$ & $24 \%$ & $27 \%$ & \\
\hline CABG and valve* & $4 \%$ & $4 \%$ & $3 \%$ & \\
\hline CPB duration (min) & $129 \pm 44$ & $129 \pm 44$ & $130 \pm 46$ & .8819 \\
\hline No. of distal bypass grafts & $3.0 \pm 1.7$ & $3.0 \pm 1.7$ & $3.2 \pm 1.6$ & .3037 \\
\hline Units of pRBCs within $48 \mathrm{~h}$ & $1.1 \pm 1.9$ & $1.1 \pm 1.9$ & $1.3 \pm 1.9$ & .4153 \\
\hline Units of platelets within $48 \mathrm{~h}$ & $0.8 \pm 3.1$ & $0.6 \pm 2.4$ & $1.9 \pm 5.7$ & .1627 \\
\hline Units of FFP within $48 \mathrm{~h}$ & $0.3 \pm 1.0$ & $0.3 \pm 1.0$ & $0.4 \pm 1.1$ & .3957 \\
\hline Any blood product within $48 \mathrm{~h}$ & $41 \%$ & $40 \%$ & $44 \%$ & .5732 \\
\hline Postoperative ICU length of stay (d) & $3.0 \pm 4.2$ & $3.0 \pm 4.1$ & $3.4 \pm 5.3$ & .0776 \\
\hline Postoperative hospital length of stay (d) & $12.0 \pm 18.1$ & $11.9 \pm 18.3$ & $12.9 \pm 17.3$ & .0284 \\
\hline
\end{tabular}

Data are presented as mean \pm SD as appropriate. Percentages presented are of the total, ie, all 466 patients. $P$ values are from the Wilcoxon rank sum test (continuous measures) or the Pearson $\chi^{2}$ test (categories) for group differences. PF4/heparin, Preoperative serum anti-platelet factor $4 /$ heparin immunoglobulin $\mathrm{G}$ level; $C A B G$, coronary artery bypass graft surgery; $C P B$, cardiopulmonary bypass; $p R B C$, allogenic packed red blood cells; $F F P$, fresh frozen plasma; $I C U$, intensive care unit. *Valve repair or replacement surgery. $\dagger$ Refers to 3-group comparison for procedure type.

ranged from 3 to 108 days, with a mean of 10.9 days (SD, \pm 13.8 days; median, 7 days). The hospital length of stay was statistically significantly longer in patients with an increased antibody result (Table 2). Deaths were associated with the following potential etiologies: sepsis/multisystem organ failure $(\mathrm{n}=8)$, ventricular failure/cardiogenic shock $(\mathrm{n}=7)$, stroke/coma $(\mathrm{n}=3)$, ischemic bowel $(\mathrm{n}=2)$, sudden death/unknown $(\mathrm{n}=2)$, and ventricular arrhythmia $(\mathrm{n}=1)$.

The relationship of Parsonnet risk score with preoperative serum anti-PF4/heparin antibody levels was not significant $(P>.75)$. Figure 1 presents the percentage of patients with a major complication by preoperative serum anti-PF4/ heparin antibody level. Of note, death or prolonged hospitalization occurred in $33.9 \%$ of patients with a positive preoperative serum anti-PF4/heparin antibody result. In

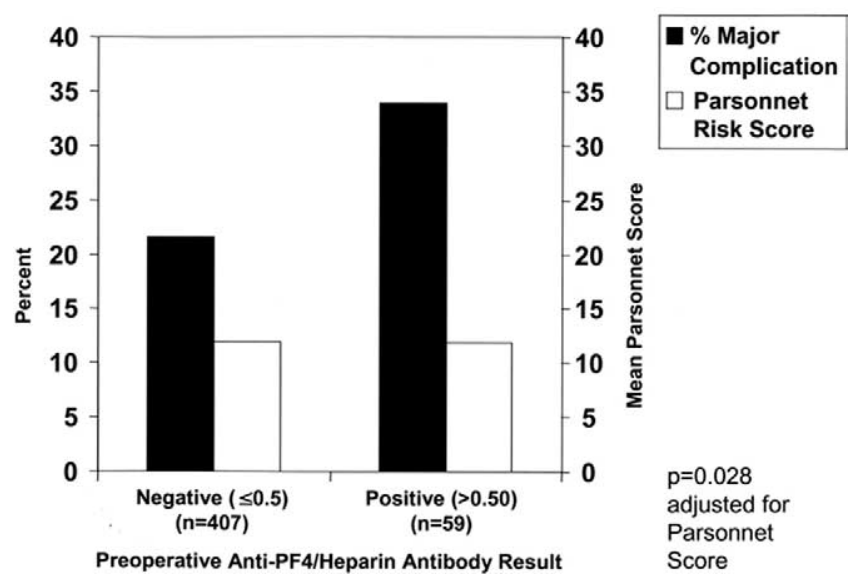

Figure 1. Major complication rate and mean Parsonnet risk score by preoperative serum anti-heparin/PF4 antibody result. contrast, $21.6 \%$ of patients with a negative assay result experienced a major complication. This difference in outcome is not explained by the Parsonnet index of known preoperative risk factors, because Parsonnet scores (mean \pm SD) were almost identical in groups with a positive versus negative assay result $(11.8 \pm 8.0$ vs $11.9 \pm 8.4 ; P=.89$; Figure 1). The logistic regression models described below help us to understand the relationships depicted in Figure 1.

We first tested our specific hypothesis with a multivariable logistic regression model that included the preoperative serum anti-PF4/heparin antibody category and the Parsonnet risk score. In this model, a positive preoperative serum anti-PF4/heparin antibody result independently predicted an increased risk for major complications $(P=.0284$; odds ratio $[\mathrm{OR}], 1.98$; 95\% confidence interval [CI], 1.06-3.62) over and above the effect of Parsonnet risk score $(P<.001$; OR, 1.07; 95\% CI, 1.05-1.10). The overall model $\chi^{2}$ for covariates (likelihood ratio test) was 32.88 with $2 d f(P<.001)$. There was no significant interaction between the preoperative serum anti$\mathrm{PF} 4 /$ heparin antibody result and the Parsonnet risk score.

To define further the validity of this model, we tested the effect of the preoperative serum anti-PF4/heparin antibody level as a continuous rather than a categorical variable. In this logistic regression model, the preoperative serum anti$\mathrm{PF} 4 /$ heparin antibody level was again independently associated with an increased risk of major complications $(P=$ .036; OR, 1.31; 95\% CI, 1.02-1.68) over and above the effect of Parsonnet risk score $(P<.001 ;$ OR, $1.07 ; 95 \%$ CI, 1.05-1.10). The overall model $\chi^{2}$ for covariates was 32.7 with $2 d f(P<.0001)$.

Three of the potential predictors from the list in Table 1 showed significant associations with major complications in separate models after adjustment for Parsonnet risk score: age $(P=.0511)$, ejection fraction $(P=.0050)$, and preop- 


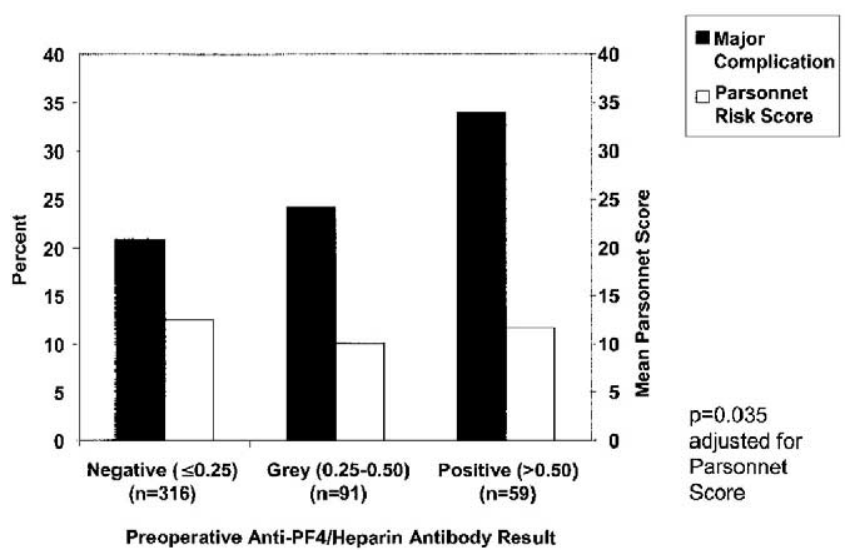

Figure 2. Major complication and mean Parsonnet risk score by preoperative serum anti-heparin/PF4 antibody result.

erative hematocrit $(P=.0432)$. (Age was marginally significant as an additional factor, even though it is represented in the Parsonnet risk score as well.) None of these 3 predictors was significantly different between the 2 antibody result groups (Table 1). When added to the model by using the binary antibody result (normal vs abnormal) and Parsonnet risk score, these 3 covariates reduced the significance of the preoperative serum anti-PF4/heparin antibody result $(P=.0889)$. However, the addition of these 3 covariates to the model by using the antibody result as a continuous variable and the Parsonnet risk score did not supplant the significance of preoperative serum anti-PF4/heparin antibody levels $(P=.0383)$.

Some investigators have characterized the anti-PF4/heparin antibodies assayed with the ELISA we used into 3 categories: positive ( $>0.5$ OD units), intermediate (0.25-0.5 OD units), and negative ( $<0.25$ OD units). ${ }^{10}$ For purposes of comparison with these data, we assessed the anti-PF4/ heparin antibodies by using a similar approach (Figure 2). Inclusion of these 3 categories of antibody, instead of the 2-category approach described previously, into multivariate analyses with Parsonnet risk score still resulted in significance of preoperative serum anti-PF4/heparin antibody levels $(P=.0349)$ as a predictor for death or prolonged hospitalization.

\section{Discussion}

This report represents the largest descriptive prospective investigation evaluating the association between preoperative anti-PF4/heparin antibodies and death or prolonged hospitalization after cardiac surgery. We have demonstrated that preoperative anti-PF4/heparin antibodies are associated with adverse outcomes after cardiac surgery and may account for a significant proportion of the variability in out- come previously reported among patients with seemingly identical preoperative risk factors.

Several prospective studies have assessed the incidence of HIT after cardiac surgery. ${ }^{9,12,17}$ Few studies have reported the prevalence of anti-PF4/heparin antibodies before cardiac surgery. The prevalence of anti-heparin/PF4 antibodies identified in our investigation, 13\%, is consistent with that in previously published reports. ${ }^{10,11}$ For example, Bauer and colleagues ${ }^{10}$ measured anti-PF4/heparin antibodies by ELISA before surgery and 5 days after surgery in 111 patients undergoing cardiac surgery with cardiopulmonary bypass. The observed prevalence of anti-heparin/PF4 antibodies was $51 \%$ after surgery versus $19 \%$ before surgery. Bauer and colleagues' study was not powered adequately to assess for the relationship between antibodies and adverse outcome. Indeed, the only outcome assessed was thrombosis. In contrast, our study assessed a more general metric of complications. Several additional similarities between these 2 studies' results are evident. In both studies, nonCaucasians were more likely to have a positive antibody assay. In addition, both studies observed a lower prevalence of anti-PF4/heparin antibodies in patients who received heparin immediately before surgery. The clinical relevance and mechanisms underlying these findings remain unclear.

The selection of our primary end point merits discussion. Prior investigations related to anti-PF4/heparin antibodies have focused on overt manifestations of HIT, namely, thrombocytopenia, thrombosis, or both-serious but relatively infrequent sequelae of cardiac surgery. Because we did not focus on HIT as an outcome, it was not rigorously assessed. Instead, our investigation assessed the association between preexisting anti-PF4/heparin antibodies and a broader measure of morbidity, ie, death or prolonged hospitalization. By the nature of its observational design, our investigation cannot define the mechanism by which heparin/PF4 antibody complexes might contribute to postoperative morbidity, mortality, or both. Nevertheless, several lines of evidence suggest that the associations we report are not spurious.

Our overall results are consistent with a recent study by Williams and associates ${ }^{18}$ that involved 218 patients with acute coronary syndromes. In their study, patients with anti-PF4/heparin antibodies were more likely to die (30.4\% vs $11.3 \% ; P=.011)$ or experience myocardial infarction (21.7\% vs $6.2 \% ; P=.008$ ). This study by Williams and associates, in conjunction with our findings, suggests a role for anti-PF4/heparin antibodies in predicting or causing adverse outcomes not classically viewed as being associated with $\operatorname{HIT}(\mathrm{T})$.

A limitation of this investigation is that anti-PF4/heparin antibodies may not be mechanistically involved in postoperative morbidity and mortality but may merely represent a surrogate marker for sicker patients at greater risk for adverse outcomes. In an attempt to minimize this confounder, 
we incorporated risk adjustment into the analyses by using a previously validated preoperative risk scoring system, the Parsonnet score, to adjust for patient-specific preoperative risk. The fact that preoperative measurement of antiheparin/PF4 antibodies independently predicted increased risk for in-hospital death or prolonged hospitalization supports the potential clinical relevance of these antibodies. Nevertheless, on the basis of our data, we cannot recommend at this time that physicians routinely test for anti-PF4/ heparin antibodies before cardiac surgery.

The mechanism(s) that could account for the adverse effects of the PF4/heparin antibodies will require further investigation. However, recent studies have demonstrated that platelets are activated by the binding of the PF4/heparin antibodies and that the $\mathrm{Fc} \gamma \mathrm{R}$ receptor plays a role in triggering the activation response. ${ }^{19,20}$ Platelets alone or in combination with leukocytes, fibrin, and other inflammatory substances could disrupt vascular function and impair postoperative recovery. The PF4/heparin antibodies can bind to endothelial cells and trigger venous and arterial thrombosis and alter organ function. Clearly, a host of factors-some genetic and others environmental—are likely to contribute to the clinical outcome in patients with these antibodies. Because these antibodies are associated with adverse outcomes, future studies should investigate whether alternative forms of intraoperative and postoperative anticoagulation, antiplatelet therapy, or both, could be beneficial. There may be a need to develop additional approaches to manage high-risk patients who have increased anti-PF4/heparin antibodies.

\section{References}

1. Mangano DT. Aspirin and mortality from coronary bypass surgery. N Engl J Med. 2002;347:1309-17.

2. Walls JT, Curtis JJ, Silver D, Boley TM, Schmaltz RA, Nawarawong W. Heparin-induced thrombocytopenia in open heart surgical patients: sequelae of late recognition. Ann Thorac Surg. 1992;53:787-91.

3. Bernard GR, Vincent JL, Laterre PF, et al. Efficacy and safety of recombinant human activated protein $\mathrm{C}$ for severe sepsis. $N$ Engl J Med. 2001;344:699-709.
4. Cella G, Vittadello O, Gallucci V, Girolami A. The release of betathromboglobulin and platelet factor 4 during extracorporeal circulation for open heart surgery. Eur J Clin Invest. 1981;11:165-9.

5. Slaughter TF, Greenberg CS. Heparin-associated thrombocytopenia and thrombosis: implications for perioperative management. Anesthesiology. 1997;87:667-75.

6. Warkentin TE, Kelton JG. Temporal aspects of heparin-induced thrombocytopenia. N Engl J Med. 2001;344:1286-92.

7. Chong BH. Heparin-induced thrombocytopenia. Br J Haematol. 1995; 89:431-9.

8. Francis JL, Palmer GJ III, Moroose R, Drexler A. Comparison of bovine and porcine heparin in heparin antibody formation after cardiac surgery. Ann Thorac Surg. 2003;75:17-22.

9. Pouplard C, May MA, Iochmann S, et al. Antibodies to platelet factor 4-heparin after cardiopulmonary bypass in patients anticoagulated with unfractionated heparin or a low-molecular-weight heparin: clinical implications for heparin-induced thrombocytopenia. Circulation. 1999;99:2530-6.

10. Bauer TL, Arepally G, Konkle BA, et al. Prevalence of heparinassociated antibodies without thrombosis in patients undergoing cardiopulmonary bypass surgery. Circulation. 1997;95:1242-6.

11. Visentin GP, Malik M, Cyganiak KA, Aster RH. Patients treated with unfractionated heparin during open heart surgery are at high risk to form antibodies reactive with heparin:platelet factor 4 complexes. J Lab Clin Med. 1996;128:376-83.

12. Trossaert M, Gaillard A, Commin PL, Amiral J, Vissac AM, Fressinaud E. High incidence of anti-heparin/platelet factor 4 antibodies after cardiopulmonary bypass surgery. Br J Haematol. 1998;101: 653-5.

13. Lindhoff-Last E, Gerdsen F, Ackermann H, Bauersachs R. Determination of heparin-platelet factor 4-IgG antibodies improves diagnosis of heparininduced thrombocytopenia. Br J Haematol. 2001;113:886-90.

14. Parsonnet V, Dean D, Bernstein AD. A method of uniform stratification of risk for evaluating the results of surgery in acquired adult heart disease. Circulation. 1989;79:I3-12.

15. Bennett-Guerrero E, Ayuso L, Hamilton-Davies C, et al. Relationship of preoperative antiendotoxin core antibodies and adverse outcomes following cardiac surgery. JAMA. 1997;277:646-50.

16. Welsby IJ, Bennett-Guerrero E, Atwell D, et al. The association of complication type with mortality and prolonged stay after cardiac surgery with cardiopulmonary bypass. Anesth Analg. 2002;94:1072-8.

17. Warkentin TE, Sheppard JA, Horsewood P, Simpson PJ, Moore JC, Kelton JG. Impact of the patient population on the risk for heparininduced thrombocytopenia. Blood. 2000;96:1703-8.

18. Williams RT, Damaraju LV, Mascelli MA, et al. Anti-platelet factor 4/heparin antibodies: an independent predictor of 30-day myocardial infarction after acute coronary ischemic syndromes. Circulation. 2003; 107:2307-12.

19. Chong BH. Heparin-induced thrombocytopenia. J Thromb Haemost. 2003;1:1471-8.

20. Newman PM, Chong BH. Heparin-induced thrombocytopenia: new evidence for the dynamic binding of purified anti-PF4-heparin antibodies to platelets and the resultant platelet activation. Blood. 2000; 96:182-7. 